\title{
Kinetic features of sliding walk in Nogaku
}

\author{
Shinsuke Yoshioka, Kazuhiro Ueda, Yui Morita, Toshio Morita, Senshi Fukashiro
}

Objectives: To investigate the kinetic features of sliding walk in Nogaku.

Design: Biomechanical motion analysis study.

Methods: Three male Nogaku actors participated in the experiment. Each actor performed the sliding walk on an 8-m walking track three times. To obtain the kinematics and kinetics, a three-dimensional optical motion capture system and two force platforms were used. The angles, moments, powers and work of each of the three lower extremity joints (i.e., hip, knee, and ankle joints) in the sagittal plane were calculated using an inverse dynamics method.

Results: The kinematic and kinetic results were successfully obtained. In the results for the vertical ground reaction force, one cycle could be clearly divided into two phases (stance and foot-sliding phases). The push force during the footsliding phase reached $53 \mathrm{~N}$ on average. The energy dissipation by the push force (friction energy dissipation) reached 14 $\mathrm{J}$, which was comparable to the hip joint work (19.7 J).

Conclusion: The following three findings were revealed: (1) The push force during the foot-sliding phase was large (average $53 \mathrm{~N}$ ). (2) The role of the hip joint was to propel the actor's body forward. The role of the knee and ankle joints was to keep the center of gravity of the actor's body low. (3) The differences in kinetic results among the actors were larger than the differences in kinematic results.

(Journal of Trainology 2012;1:10-13)

Key words: Japanese theatrical art $\mathbf{\square}$ kinetic analysis $\mathbf{a}$ friction force

$\mathrm{N}$ gaku, ${ }^{1,2}$ a theatrical art unique to Japan, is the oldest performing art in the world and is one of the intangible cultural heritages listed by UNESCO. To help further the understanding and preservation of Nogaku, Hachimura and colleagues have measured the dance motions made by Nogaku performers and archived them digitally. ${ }^{3}$ However, the archive focuses only on the outward appearance of each dance motion. To understand Nogaku, evaluating performer sensation is also important. However, objective data on performer sensation is lacking in Nogaku research. For that reason, we conducted a kinetic analysis of Nogaku, since kinetic parameters, such as ground reaction force and joint moment, relate to the performer's skin and muscle sensations. For example, ground reaction force is related to the skin sensation of a foot sole.

The most basic posture in Nogaku is called Kamae. An actor in this posture bends his knees slightly to keep his center of gravity (CG) low. The most basic locomotive movement is called Hakobi (Figure 1a and 1b). In this movement, an actor does not lift his feet but slides them forward while keeping his upper body in the Kamae posture and his CG low. Because Hakobi comprises only a few body movements, the forward motion of the actor's body is hardly perceptible. The body of an actor performing Hakobi is thus sometimes paradoxically called a "still body," in contrast with the "acrobatic bodies" seen in many plays and dances, such as ballet in Europe,
Beijing opera in China, and samul nori in Korea. ${ }^{4}$

The purpose of this study was to investigate the kinetic features of Hakobi, the basic locomotive movement in Nogaku.

\section{Methods}

Three male Nogaku actors gave informed consent to participate in the experiment. This study was approved by the ethical committee of the University of Tokyo. The age, height, and body mass of the three actors were 31 years, $1.69 \mathrm{~m}$, and $85.3 \mathrm{~kg}$ for Actor 1, 29 years, $1.70 \mathrm{~m}$, and $75.0 \mathrm{~kg}$ for Actor 2, and 49 years, $1.70 \mathrm{~m}$, and $71.9 \mathrm{~kg}$ for Actor 3. Actor 1 made his debut at the age of 5 years, with a career spanning 26 years. Actor 2 made his debut at the age of 6 years, with a career spanning 23 years. Actor 3 made his debut at the age of 26 years, with a career spanning 23 years. All actors wore socks for the experiment, as they do on stage when acting in actual Nogaku performances. Each actor performed Hakobi three times in the typical manner and at the standard speed. The walking track for this experiment was 8 meters long. The data from only two of the three trials were analyzed since deficits were found in the data from one of the trials.

To obtain the kinematics, three-dimensional coordinates of landmark points on the body were acquired at $200 \mathrm{~Hz}$ using a

Received February 9, 2012; accepted March 1, 2012

From the Faculty of Sport and Health Science, Ritsumeikan University, Kusatsu, Shiga, Japan (S.Y.), Interfaculty Initiative in Information Studies, The University of Tokyo \& CREST, Japan Science and Technology Agency, Komaba, Tokyo, Japan (K.U.), NPO Association for Education of Japanese Traditional Performing Arts, Denenchofu, Tokyo, Japan (Y.M.), Department of Mechanical Engineering, Keio University, Hiyoshi, Yokohama, Japan (T.M.), and Graduate School of Interdisciplinary Studies, The University of Tokyo, Komaba, Tokyo, Japan (S.F.).

Communicated by Takashi Abe, $\mathrm{PhD}$

Correspondence to Professor Senshi Fukashiro, Ph.D., Sport Biomechanics Laboratory, Department of Life Sciences, Graduate School of Interdisciplinary Studies, The University of Tokyo, 3-8-1 Komaba, Meguro-ku, Tokyo 153-8902, Japan. E-mail: fukashiro@idaten.c.u-tokyo.ac.jp

Journal of Trainology 2012;1:10-13 @2012 The Active Aging Research Center http://trainology.org/ 

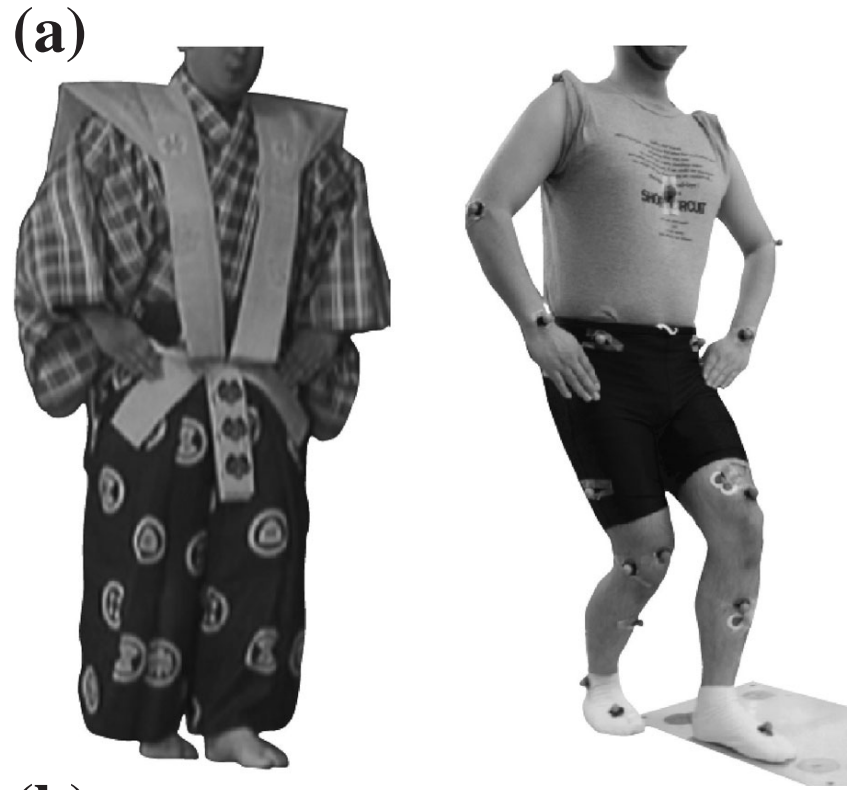

(b)

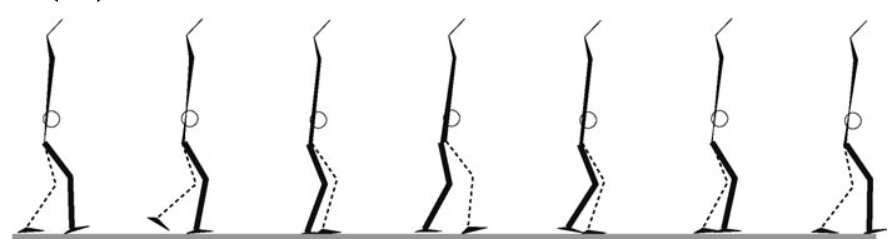

Figure 1. (a) The figure on the left shows an actor performing Hakobi on an actual stage. (This picture is taken from the DVD entitled "The Kyogen of the Tojiro Yamamoto Kyogen Company," with permission of the Japan Traditional Cultures Foundation, the distributor of the DVD.) The figure on the right shows an actor performing Hakobi in our experimental setting, with 30 markers (hemispherical silver objects) placed on his body to track his movements. (b) Stick figures depict body movement during Hakobi and show a typical single cycle from one heel contact to the next. The open circles show the center of gravity of the actor's body mass.

three-dimensional optical motion capture system with seven cameras (Hawk Digital System, Motion Analysis Corporation, Santa Rosa, CA, USA). Thirty reflective markers were placed on the body (head x 3 , scapula x 1 , sternum x 1 , shoulder x 2 , elbow x 2, wrist x 2, anterior superior iliac spine $\mathrm{x} 2$, sacrum $\mathrm{x}$ 1 , great trochanter $\times 2$, thigh $\times 2$, knee $\times 2$, patella $\times 2$, shank $x$ 2 , ankle $\mathrm{x} 2$, heel $\mathrm{x} 2$, and toe $\mathrm{x} 2$ ). The ground reaction force was measured at $1000 \mathrm{~Hz}$ using two force platforms (9281B, Kistler Instruments AG, Winterthur, Switzerland) placed under the center of the walking track.

The raw coordinates data were smoothed using a fourthorder zero phase shift Butterworth low-pass filter at the cutoff frequency of $6 \mathrm{~Hz}$. The cutoff frequency was determined by conducting a residual analysis. ${ }^{5}$ Using the data and assuming each actor's body as a link segment model, we calculated the angles of the three lower extremity joints (the hip, knee, and ankle joints) in the sagittal plane. ${ }^{6}$ We also calculated the moments and power of these joints as follows: The equations of translational and rotational motion of each segment were used to calculate joint reaction forces and moments by using an inverse dynamics method. ${ }^{5}$ The equations for the feet, legs, thighs, and HAT (head, arms, and trunk) were used to calculate the joint reaction force and moments at the ankle, knee, and hip joints. The mass of each segment and the moment of inertia values about the transverse axis of each segment reported by de Leva were used. ${ }^{7}$ Joint power was calculated by multiplying joint moment by joint angular velocity. Joint angular velocity was calculated by differentiating the joint angle with respect to time. The work done by each joint during one cycle was calculated by integrating the power with respect to time. Energy dissipation resulting from the friction between foot and floor during the foot-sliding phase was calculated using the horizontal ground reaction force and the horizontal displacement of the toe marker.

\section{Results}

The profiles of the CG height, joint angle, moment, and power for all of the actors were similar in both trials (Figures 2, 4, and 5). The CG height during Hakobi was always lower

(a)
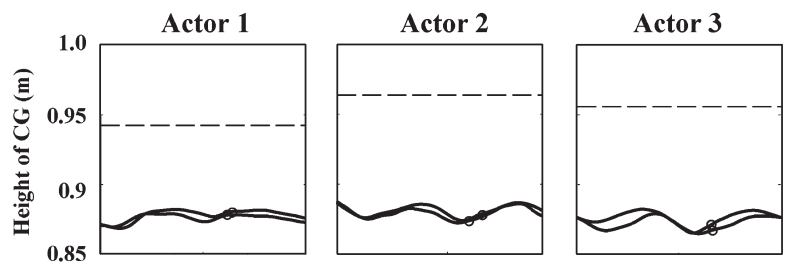

(b)
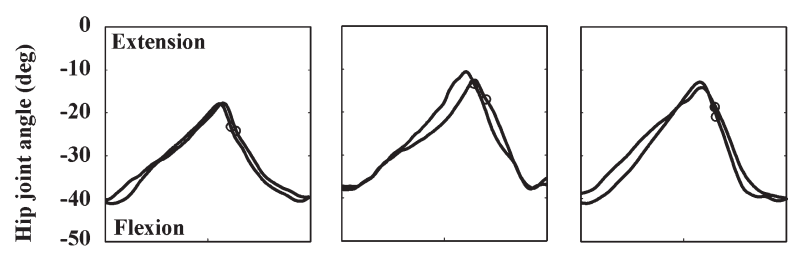

(c)
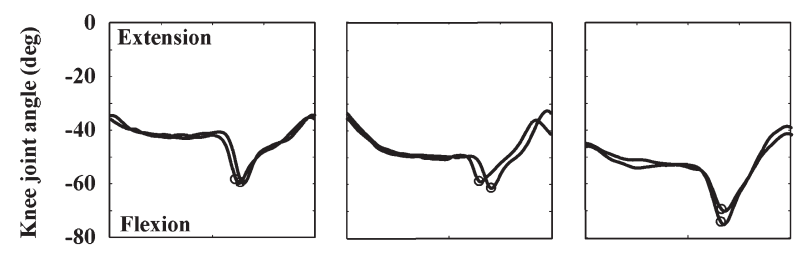

(d)
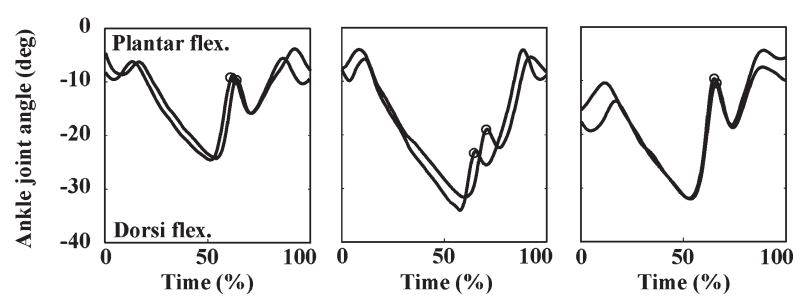

Figure 2. Kinematics during Hakobi: (a) time course of CG height, (b) time course of hip joint, (c) time course of knee joint, and (d) time course of ankle joint. The open circles in each graph show the transition time between the stance and foot-sliding phases, with each horizontal axis showing time as a percentage of the time needed to complete one cycle. The broken lines in (a) show the CG height in a static upright standing position. The hip and knee extension angles and ankle plantar flexion angle were defined as positive. 
(a)

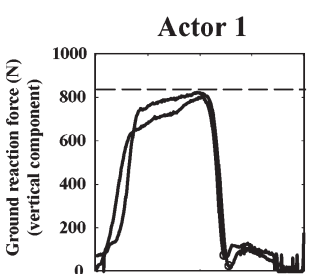

(b)

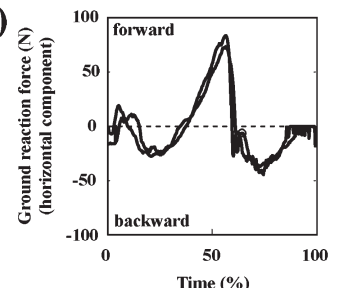

Actor 2
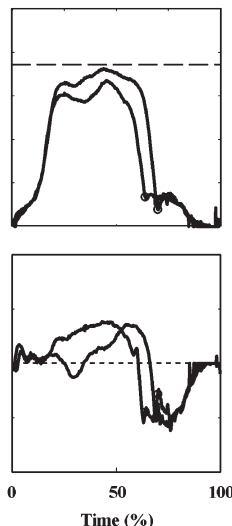

Actor 3
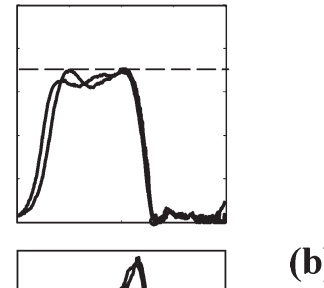

Figure 3. Ground reaction force during Hakobi: (a) vertical component and (b) horizontal component. The open circles in each graph show the transition time between the stance and foot-sliding phases, with each horizontal axis showing time as a percentage of the time needed to complete one cycle. The broken lines in (a) show the body weight of each actor.

(a)

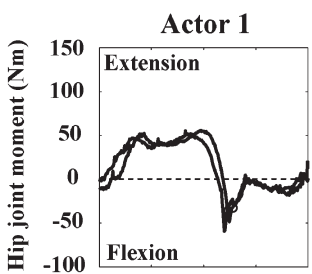

(b)

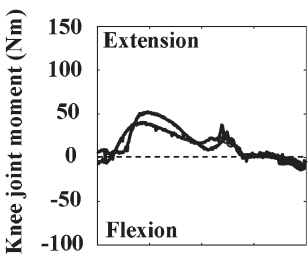

(c)

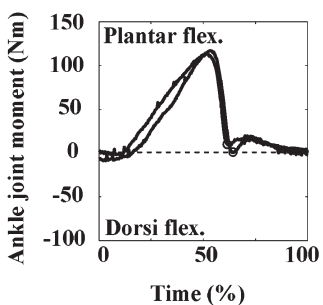

Actor 2
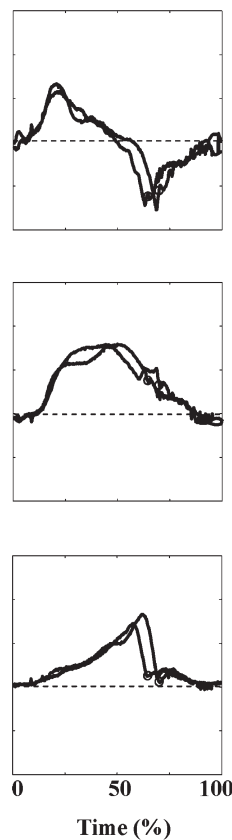

Actor 3
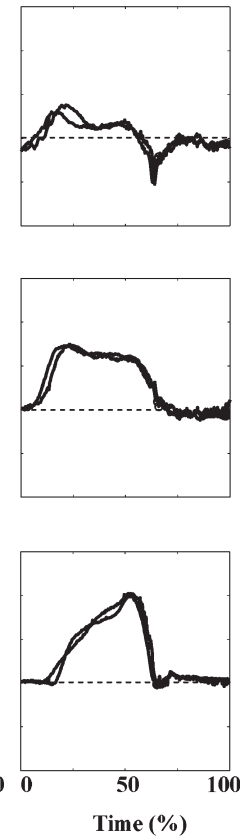

Figure 4. Joint moment during Hakobi: (a) hip joint, (b) knee joint, and (c) ankle joint. The open circles in each graph show the transition time between the stance and foot-sliding phases, with each horizontal axis showing time as a percentage of the time needed to complete one cycle. The hip and knee extension moments and ankle plantar flexion moment were defined as positive.

than that in the static upright standing position (average: 7.7 $\mathrm{cm}$ ). The vertical displacement of $\mathrm{CG}$ was very small (average: $1.4 \mathrm{~cm}$ ). Also, the profiles of the hip, knee, and ankle joint angles were similar for all three actors (Figure 2b-d). The knee joint angle remained almost constant for the entire time, ranging from $0 \%$ to $50 \%$ (Figure 2c). The average velocity of Hakobi was $0.50 \mathrm{~m} / \mathrm{s}$.

(a)

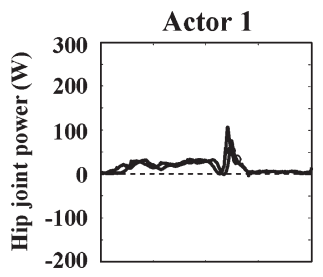

Actor 2

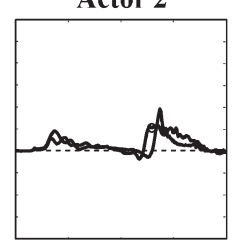

Actor 3

(b)
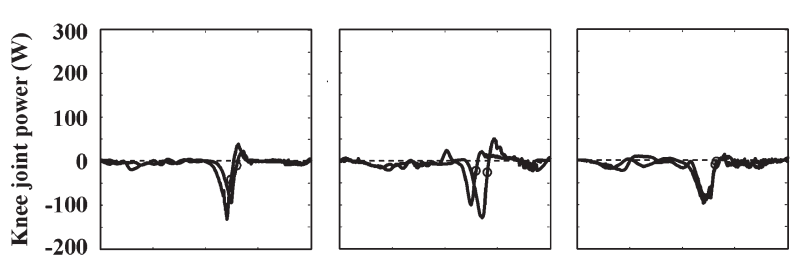

(c)
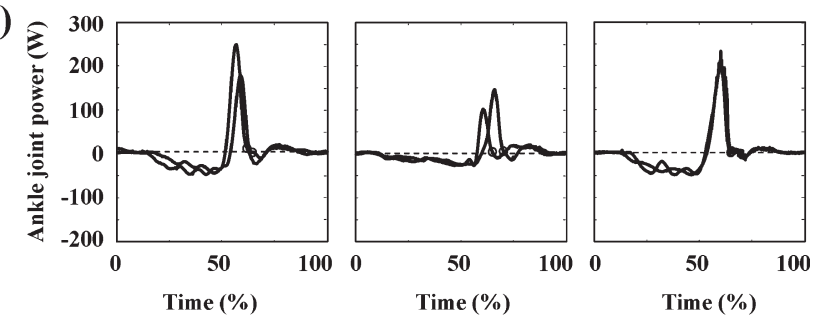

Figure 5. Joint power during Hakobi: (a) hip joint, (b) knee joint, and (c) ankle joint. The open circles in each graph show the transition time between the stance and foot-sliding phases, with each horizontal axis showing time as a percentage of the time needed to complete one cycle.

In the vertical ground reaction force results, one cycle could be clearly divided into two phases (stance and foot-sliding phases) (Figure 3a). The transition points are depicted in Figures 2-5 with open circles. During the stance phase, the vertical ground reaction force remained almost constant, and the values were almost equivalent to the actor's body weight (broken line in Figure 3a). During the foot-sliding phase, each actor pushed his foot forward and downward on the floor (Figure 3). However, the push force used by Actor 3 (average: $27 \mathrm{~N}$ ) was far smaller than that used by Actor 1 (average: 62 $\mathrm{N})$ and Actor 2 (average: $71 \mathrm{~N}$ ).

The profiles of the hip and knee joint moments were different for all three actors (Figure 4a-b), whereas the profiles of the ankle joint moments were similar (Figure 4c). The hip, knee, and ankle joint moments during the foot-sliding phase of Actors 1 and 2 were slightly larger than those during the footsliding phase of Actor 3.

The profiles of the hip, knee, and ankle joint powers were similar for the three actors (Figure 5a-c). The hip and knee joint powers were positive and negative, respectively, throughout one cycle. The ankle joint power was negative in the early part of the stance phase, and then became positive during the last half. The peak ankle joint power was the largest. The average hip, knee, and ankle joint work during one cycle was 19.7, -10.6, and $0.6 \mathrm{~J}$, respectively. The average total friction energy dissipating throughout the foot-sliding phase was $14.0 \mathrm{~J}$. 


\section{Discussion}

The purpose of this study was to investigate the kinetic features of Hakobi. The most distinguishing result in our kinetic analysis was the large push force during the footsliding phase (average: $53 \mathrm{~N}$ ) (Figure 3). The energy dissipation by this force (friction energy dissipation) reached $14 \mathrm{~J}$, which was comparable with the hip joint work (19.7 J). When actors practice Hakobi, they are taught by their master with the following words: "Concentrate on your center." It is thought that the large ground reaction force is a result of this kind of training. Additionally, it was found that the push forces of Actors 1 and 2 were more than two times larger than that of Actor 3. Because it is recommended that actors begin the practice of Japanese theatrical arts, such as Nogaku, Bunraku, and Kabuki, in early childhood, ${ }^{9}$ it is suggested that the difference in push force is due to the difference in the age at which the actors started their training (Actors 1 and 2: 5 and 6 years, respectively; Actor 3: 20 years). These findings could not be determined solely from kinematics or qualitative observation, since Hakobi is not a rational motion from a biomechanical perspective in terms of such things as as efficiency and mobility. In other words, this finding is unique to a study that uses a force platform-namely, this study.

The hip joint exerted positive power throughout one cycle, and the hip joint work was the largest among the three joints. These results indicate that the role of the hip joint is to propel the actor's body forward. The knee joint exerted large and relatively constant moment during the stance phase. However, the joint power was nearly zero or negative during this phase. These results indicate that the role of the knee joint is to keep the CG of the actor's body low. The ankle joint exerted the largest moment of the three joints. This is attributed to the location of the ankle joint, since the mass existing above the ankle joint is the largest of the three joints. Also, the joint work during one cycle was nearly zero. These results indicate that the role of the ankle joint, like that of the knee joint, is to keep the CG of the actor's body low.

Among the three actors, the differences in the kinetic results, such as the ground reaction force during the foot-sliding phase and the hip and knee joint moments, were larger than the differences in the kinematic results, such as the CG height and joint angle (Figure 2, 3, and 4). These results show that kinetic parameters represent characteristics of each actor better than the kinematic ones. In other words, if we wish to understand the characteristics of each actor, we should focus not only on kinematics but also on kinetics. Furthermore, if we wish to shed light on the key points of Hakobi motion as performed by a master of Nogaku, this finding is very useful. This finding also applies to archiving Nogaku digitally. The results of our study suggest that kinetics can contribute to the training of actors in Hakobi as well as reveal the differences between a less experienced actor and a more skillful one. These kinds of objective data can help an actor efficiently improve his skill in Hakobi.

\section{Conclusion}

The following three findings were revealed: (1) The push force during the foot-sliding phase was large. (2) The role of the hip joint was to propel the actor's body forward, while the role of the knee and ankle joints was to keep the CG of the actor's body low. (3) The differences in the kinetic results among the actors were larger than the differences in kinematic results.

\section{Acknowledgements}

This project was supported by the Ministry of Education, Culture, Sports, Science and Technology in Japan (No: 21650155) and by a Toray Science and Technology Grant. We thank Norishige Yamamoto, Norihide Yamamoto, and Takashi Wakamatsu, all of whom are Kyogen actors of the Yamamoto family in the Ohkura School for permitting us to measure their Hakobi walking.

\section{References}

1. Komparu K. The Noh Theater: Principles and Perspectives. Warren, CT: Floating World Editions; 2005.

2. The Japan Arts Council. Noh and Kyogen: An Introduction to Noh and Kyogen. http://www2.ntj.jac.go.jp/unesco/noh/en/index.html. Accessed January 31, 2011.

3. Hachimura K. Digital Archiving of Dancing. NCD Review 2006; 8: 51-66.

4. Matsuoka S. Utage-no-shintai (Body at the Banquet) (in Japanese). Tokyo, Japan: Iwanami Shoten; 2004.

5. Winter DA. Biomechanics and Motor Control of Human Movement. 4th ed. Hoboken, NJ: John Wiley and Sons, Inc.; 2009.

6. Yoshioka S, Nagano A, Himeno R, et al. Computation of the kinematics and the minimum peak joint moments of sit-to-stand movements. Biomed Eng Online 2007. doi: 10.1186/1475-925X-6-26.

7. de Leva P. Adjustments to Zatsiorsky-Seluyanov's segment inertia parameters. J Biomech 1996; 29: 1223-1230.

8. Nomura M. Kyougen = Human Comedy (in Japanese). Teaching English Now 2009; 15: 1. Tokyo, Japan: SANSEIDO; 2009. http://tb.sanseido.co. jp/english/newcrown/pdf/ten015/ten_vol15_01.pdf. Accessed February 20, 2011.

9. Shibata S. Gigei-no-syuuren to zyukutatsu-no-mechanism Kabuki-nogeidan wo tsuuran-shite (in Japanese). Studies in Language and Culture 2010; 31: 61-82. 